\title{
Boundary integral equation formulations for free-surface flow problems in two and three dimensions*
}

\author{
J.E. Romate \\ Netherlands Technology Foundation, c/o Delft Hydraulics, P.O. Box 152, NL-8300 AD Emmeloord, The Netherlands
}

P. J. Zandbergen

University of Twente, P.O. Box 217, NL-7500 AE Enschede, The Netherlands

\begin{abstract}
To compute the transient solution of free-surface flow problems in two and three dimensions boundary integral equation formulations are considered. Consistent lower and higher order approximations based on small curvature expansions are compared and applied to a time-dependent, linear free-surface wave problem.
\end{abstract}

\section{Introduction}

In many problems in ocean engineering irrotational gravity waves play an important role. When large fixed or floating bodies are involved (i.e., bodies with a characteristic length of the order of the wave length) viscosity and compressibility effects, as well as surface tension, are of minor importance, and the fluid flow is described well by a potential flow. The velocity potential $\phi$, governing the flow, satisfies the Laplace equation

$\nabla^{2} \phi=0$

throughout the fluid domain $\Omega$. The motion of the free surface waves is described by the dynamic and the kinematic free-surface boundary conditions. These boundary conditions are nonlinear, timedependent partial differential equations

$$
\begin{aligned}
& \frac{\partial \phi}{\partial t}+\frac{1}{2}(\nabla \phi)^{2}+g \cdot \eta=f(t), \\
& \frac{\partial \eta}{\partial t}+\frac{\partial \phi}{\partial x} \cdot \frac{\partial \eta}{\partial x}+\frac{\partial \phi}{\partial y} \cdot \frac{\partial \eta}{\partial y}-\frac{\partial \phi}{\partial z}=0,
\end{aligned}
$$

where $\eta$ is the free surface elevation anf $g$ is the gravitational acceleration. The nonlinear waves described by (1)-(3) are of theoretical interest (e.g., wave-wave interaction, and the deformation of steep waves), as well as of practical interest (e.g., wave forces on offshore structures). In order to solve (1)-(3), we also need boundary conditions on all other boundaries of $\Omega$, as well as appropriate initial conditions for the time-dependent boundary conditions. Assuming that wave amplitude and body motions are small compared to wave length and water depth, boundary conditions may be linearized and moving boundaries fixed. But even with these simplifications analytical solutions only have been found for very special geometries, and numerical methods are needed to solve more general free surface problems, such as the nonlinear problem (1)-(3).

In recent years, this type of nonlinear wave problem has been solved succesfully with the use of boundary integral methods (Longuet-Higgins and Cokelet 1976; Vinje and Brevig 1981). For this reason an integral equation method has been chosen to be used in the solution of the equivalent problem in $3 \mathrm{D}$. In three dimensions the solution of (1)-(3) is much more complex than it is in two

* Enhanced version of a paper presented at the IUTAM-Symposium on advanced boundary element methods, applications in solid and fluid mechanics, April 13-16, 1987, San Antonio/Tx, USA 
dimensions. Not only is the process of solving the system of differential equations much more complex, but also problems are encountered in for instance geometric modeling, numerical grid generation and flow visualization; not to mention the fact that usually $3 \mathrm{D}$ simulations are restricted severely by computing time and storage requirements.

Therefore, we will focus our attention on the efficient computation of the solution of the Laplace equation in 3D using integral equations. Especially the choice of integral equation(s) and the analysis of the order of accuracy will be considered. Also some new results in this respect will be given. Using this analysis a versatile computer program which allows the selection of a number of different formulations, has been written. The program is modular and vectorized. It turns out to be most efficient. This will be demonstrated by considering a linear free surface wave.

Before going into details, we want to mention some of the advantages and disadvantages of the integral equation method (IEM) in comparison with field discretization methods, such as the finite element method (FEM). The fact that only the boundary of the fluid domain has to be discretized (using IEM), has some obvious advantages. In the first place, the problem of numerical grid generation is reduced to the generation of a surface grid, instead of a volume grid. A surface grid is more easy to generate, and of course also cheaper. Secondly, the reduction of the number of unknowns means easier data handling, less storage problems, and also a more simple flow visualization. These features make the integral equation method suited very well for problems involving moving boundaries.

The disadvantages are also clear. The IEM is rather complex, and therefore difficult to program, it is sensitive to the discretization, and later extensions to solve other equations than the Laplace equation are difficult or impossible.

As far as the computational effort of IEM and FEM is concerned, it is less apparent which of the methods is the more attractive. To obtain some idea of the effort involved, consider a rectangular box $\Omega$, which is discretized for FEM resulting in a regular $(\alpha n \times n \times n)$-grid, where $\alpha n$ is an integer and $\alpha \leqslant 1$. This grid also defines the surface grid for the integral equation method. The most timeconsuming parts in the calculations of both methods are the calculation of the matrix coefficients $A_{i j}$, and the solution of the matrix equation $A \boldsymbol{x}=\boldsymbol{b}$, and therefore only these parts will be considered.

For basic methods (involving lower order polynomials) the operation counts are given in Table 1 (neglecting small terms). The influence of the geometry, i.e., of the aspect ratio $\alpha$, is considerable. For small $\alpha$ there will be relatively few internal nodes, and integral equation methods will be favourable only for very large $n$. Example: Suppose $c_{i}=20$, that is, it takes 20 operations to calculate one matrix coefficient using an IEM (only for large $N_{i}$ (say $N_{i}>1000$ ); for small $N_{i} c_{i}$ will be $O(200)$ ). And furthermore, suppose an iterative method can be used for IEM, with $k=n$, i.e., it converges in $O\left(\sqrt{N_{i}}\right)$ iterations. Then the approximate total number of operations, for $\alpha=1.0$ and $\alpha=0.1$, are given in Table 2. It is clear that integral equation methods will be more efficient than finite element methods if $n$ is large enough.

\section{Integral equation methods}

Assume that the simply-connected domain $\Omega$ is bounded by the closed surface $S$, consisting of $C^{2}$ continuous subsurfaces $S_{i}$. Using Green's third identity a large number of equivalent integral

Table 1. Operation counts for a lower order IEM and a lower order FEM; ( $c_{i}$ and $c_{f}$ are coefficients)

\begin{tabular}{lll}
\hline & IEM & FEM \\
\hline band width $(M)$ of $A$ & full & $\alpha n^{2}+(1+2 \alpha) n$ \\
number of nodes $(N)$ & $(2+4 \alpha) n^{2}$ & $\alpha n^{3}+(1+2 \alpha) n^{2}$ \\
storage & $N_{i}^{2}$ & $N_{f} M_{f}$ \\
coefficients $A_{i j}$ & $c_{i} N_{i}^{2}$ & $c_{f} 20 N_{f}$ \\
solution $A \boldsymbol{x}=\boldsymbol{b}$ & $1 / 3 N_{i /}^{3} / k N_{i}^{2 *}$ & $1 / 2 N_{f} M_{f}^{2}$ \\
\hline
\end{tabular}

* $1 / 3 N_{i}^{3}$ for Gauss elimination, $k N_{i}^{2}$ for iterative methods 
Table 2. Number of operations for the integral equation method and the finite element for two aspect ratios $\alpha$

\begin{tabular}{llll} 
& $\operatorname{IEM}\left(K_{i}\right)$ & $\operatorname{FEM}\left(K_{f}\right)$ & $K_{i} \geqslant K_{f}$ \\
\hline$\alpha=1.0$ & $(36) n^{5}+(720) n^{4}$ & $(0.5) n^{7}+(4.5) n^{6}$ & $n \geqslant 11$ \\
$\alpha=0.1$ & $(5.8) n^{5}+(116) n^{4}$ & $(0.0005) n^{7}+(0.018) n^{6}$ & $n \geqslant 100$ \\
& & $+(0.216) n^{5}$ & \\
\hline
\end{tabular}

equation formulations can be derived to solve the Laplace equation (1) in $\Omega$. All are based on source and/or dipole distributions on the boundary $S$. In this paper two families of integral equations are considered:

$\phi(x)+\frac{1}{2} \mu(\boldsymbol{x})=f_{S} \sigma \cdot G+\mu \cdot \frac{\partial G}{\partial n} \mathrm{~d} S$
$\frac{\partial \phi}{\partial n_{x}}(\boldsymbol{x})-\frac{1}{2} \sigma(\boldsymbol{x})=\int_{S} \sigma \cdot \frac{\partial G}{\partial n_{x}}+\mu \cdot \frac{\partial^{2} G}{\partial n_{x} \partial n} \mathrm{~d} S$

with

$G(x ; \xi)=\frac{-1}{4 \pi\|x-\xi\|}$.

Here, $\xi$ is the integration variable on $S, \sigma(\xi)$ and $\mu(\xi)$ are the source and dipole distribution on $S$, and $\boldsymbol{n}_{x}$ and $\boldsymbol{n}$ denote the local inward normals at $\boldsymbol{x}$ and $\boldsymbol{\xi}$ respectively (Fig. 1). The symbol $f f_{S}$ denotes the finite part of the integral $\iint_{S}$ in the sense of Hadamard.

To make the problem determinate a mix of $\sigma$ and $\mu$ has to be chosen in (4)-(5) for each subsurface. A certain choice should render a well-posed problem, having a unique solution. Furthermore the numerical errors should be as small as possible. Three obvious choices are:
(1) $\sigma=0$,
(dipole-only)
(2) $\mu=0$
(source-only)
(3) $\sigma=\frac{\partial \phi}{\partial n}, \mu=-\phi$
(Green's identity)

Source-only distributions, dipole-only distributions and mixed distributions all take about the same computational effort. However, as Hunt (1978) states, the choice of a mix of source and dipole distributions leads to better results than a source-only or a dipole-only distribution, as it reduces leakage considerably. For this reason Green's formulation $\left(\sigma=\frac{\partial \phi}{\partial n}, \mu=-\phi\right)$ is favourable. If $\phi$ and $\nabla \phi$ are both of interest on a boundary $S$ it is also more advantageous to use Green's formulation. To illustrate this consider the free-surface boundary conditions involving both $\phi$ and $\nabla \phi$ and suppose $\phi$ is known. Then $\frac{\partial \phi}{\partial n}$ is known from the boundary integral equation method and the two tangential derivatives $\frac{\partial \phi}{\partial t_{1}}$ and $\frac{\partial \phi}{\partial t_{2}}$ can be computed easily form $\phi$, thus giving $\nabla \phi$ on $S$. Any other choice of singularities would lead to the necessity of calculating extra influence coefficients per singularity in

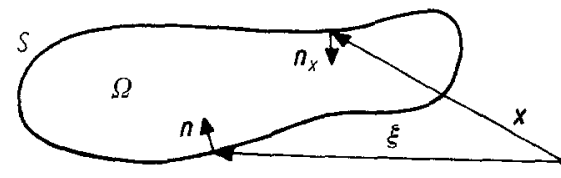

Fig. 1. Definition of geometric quantities 
Table 3. Summary of results on local errors

\begin{tabular}{|c|c|c|c|c|}
\hline $\begin{array}{l}\text { option } \\
\text { in program }\end{array}$ & $\begin{array}{l}\text { local } \\
\text { trunc. error } \varepsilon_{\Delta}\end{array}$ & $\sigma$ & $\mu$ & panel geometry \\
\hline \multicolumn{5}{|l|}{ int. Eq. (4) } \\
\hline A & $O(\Delta)$ & const. & const. & flat \\
\hline B & $O\left(\Delta^{2}\right)$ & const. & lin. & quadr. $^{\mathrm{a}}$ \\
\hline $\mathrm{C}$ & $O\left(\Delta^{3}\right)$ & lin. & quadr. & cubic $^{b}$ \\
\hline int. Eq. (5) & $O(1)$ & const. & const. & flat \\
\hline $\mathrm{D}$ & $O(\Delta)$ & const. & lin. & flat \\
\hline $\mathrm{E}$ & $O\left(\Delta^{2}\right)$ & lin. & quadr. & quadr. \\
\hline
\end{tabular}

a Integrals involving quadratic curvature terms can be neglected only if the boundary $S$ is everywhere $C^{2}$-continuous

${ }^{b}$ Integrals involving cubic curvature terms can be neglected only if the boundary $S$ is everywhere $C^{3}$-continuous; here also higher order approximations are needed for the line segments representing the panel boundaries (Romate 1988). In the program the cubic terms and curved panel boundaries are not included

order to calculate $\nabla \phi$ from the solved $\sigma$ and $\mu$. For these and some other less important reasons Green's formulation will be used.

To solve the boundary value problem numerically the boundary $S$ is discretized in $N$ quadrilateral panels. In general the integrals in (4) and (5) over a panel surface $\Delta S$ cannot be calculated analytically. Nonetheless, using truncated Taylor series to approximate the panel surface and the singularity distributions, analytical expressions can be derived approximating the integrals to a certain order in terms of the typical panel diameter $\Delta$. It is remarked that even the integral in Eq. (5) representing the velocity due to a dipole distribution on $S$, with its hypersingular kernel, is calculated analytically. (This is done by first reformulating the integral in terms of a surface vorticity distribution and a line vortex along the boundary of $S$.) A local truncation error analysis as in Hess (1975) or Romate (1988) shows which terms in each of the Taylor series should be taken into account. Table 3 shows the minimum approximations needed for the source $\sigma\left(=\frac{\partial \phi}{\partial n}\right)$, the dipole $\mu(=-\phi)$ and the panel geometry to obtain a certain order for the local truncation error $\varepsilon_{\Delta}$.

The singularity distributions are expressed in terms of the strenghts of the singularities in the $N$ centers of the panels where the boundary conditions are imposed. Applying a discretized integral equation at each collocation point $\boldsymbol{x}_{i}$ and substituting the boundary conditions will yield a set of $N$ linear equations with $N$ unknown values $\phi\left(\boldsymbol{x}_{i}\right)$ or $\frac{\partial \phi}{\partial n}\left(\boldsymbol{x}_{i}\right)$.

It is clear that the integral Eqs. (4) and (5) can be chosen such that all resulting equations are Fredholm integral equations of the second kind. For example, in case of a Dirichlet condition at $\boldsymbol{x}_{i}$ on $S$, the application of Eq. (5) for $\boldsymbol{x}=\boldsymbol{x}_{i}$, results in a Fredholm equation of the second kind for $\frac{\partial \phi}{\partial n}(\boldsymbol{x})$.

The matrix constructed this way has large coefficients on the diagonal. In general iterative methods can be used to solve the matrix equation.

\section{Asymptotic convergence}

Based on the results of the local error analysis five options were implemented in the developed computer program (see first column of Table 3). Various tests in both two and three dimensions were done to analyse the numerical behavior of the methods. The main results are given here. For smooth boundaries $S$ the global errors of the methods are of the same order as the corresponding local errors. E.g., the higher order method $E$ has a local truncation error of $O\left(\Delta^{2}\right)$ and thus the global error also is $O\left(\Delta^{2}\right)$ as $\Delta \rightarrow 0$. For non-smooth boundaries the global error will be one order lower than the corresponding local error if Eq. (4) is used for a Dirichlet problem, i.e., the global 
errors of the methods $\mathrm{A}, \mathrm{B}$ and $\mathrm{C}$ are then $O(1), O(\Delta)$ and $O\left(\Delta^{2}\right)$ respectively. This is shown in Fig. 2 , where the analytical solution $\phi$ of the flow around an infinite circular cylinder (radius 1) was imposed on the boundary of an infinite ,square cylinder". The corresponding exterior problem was then solved, and $\frac{\partial \phi}{\partial n}$ was determined on the boundary of the square cylinder using the five methods. the maximum error $\left|\frac{\partial \phi}{\partial n}-\frac{\partial \phi}{\partial n}\right|_{\infty}$ is given in Fig. 2.

In practical calculations it suffices to introduce a strip of higher order panels along the edges of the subsurfaces $S_{i}$ where slope-discontinuities may occur, to compensate the loss of accuracy. Figure 2 also shows that method D is less accurate than method B which involves approximately the same computational effort, just as the more expensive method $\mathrm{E}$ is less accurate than the comparably expensive method $\mathrm{C}$.

\section{A linear free surface problem}

We will now consider the application of the method to a linear free-surface problem, governed by the Laplace Eq. (1) and the linearized free-surface boundary conditions:

$$
\begin{array}{ll}
\frac{\partial \phi}{\partial t}+g \eta=0 & \text { on } z=0 \text { (still water level) } \\
\frac{\partial \eta}{\partial t}-\frac{\partial \phi}{\partial z}=0 & \text { on } z=0 \\
\frac{\partial \phi}{\partial n}=0 & \text { on } z=-h \text { (bottom) }
\end{array}
$$

In the numerical computations the infinite domain is truncated by artifical boundaries at some distance from the region of interest (Fig. 3).

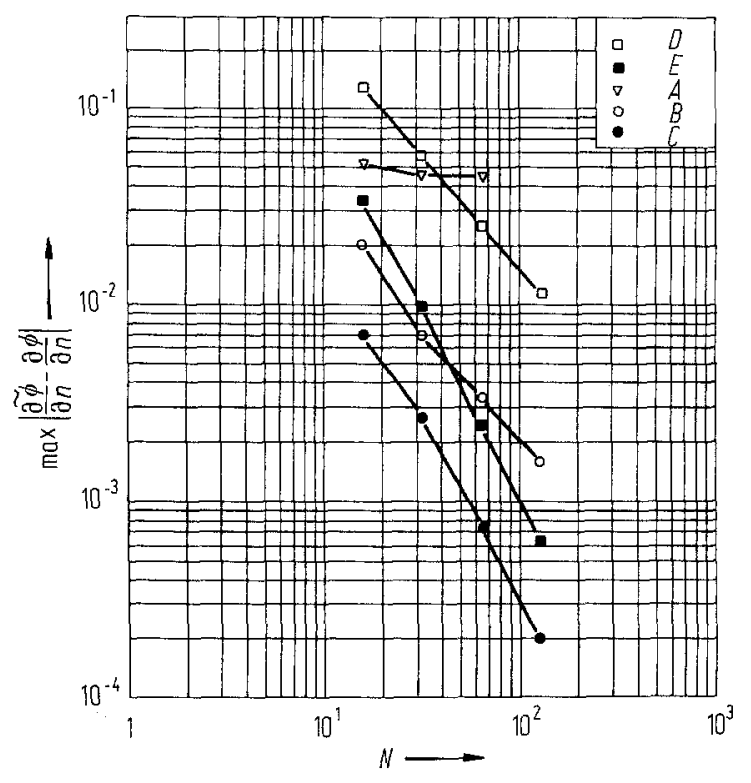

2

Figs. 2 and 3. 2 Asymptotic error behavior of the 5 methods for the "square cylinder" problem ( $N=$ number of panels in $x$ direction). 3 Rectangular fluid domain $\Omega(y=0$ is plane of symmetry)

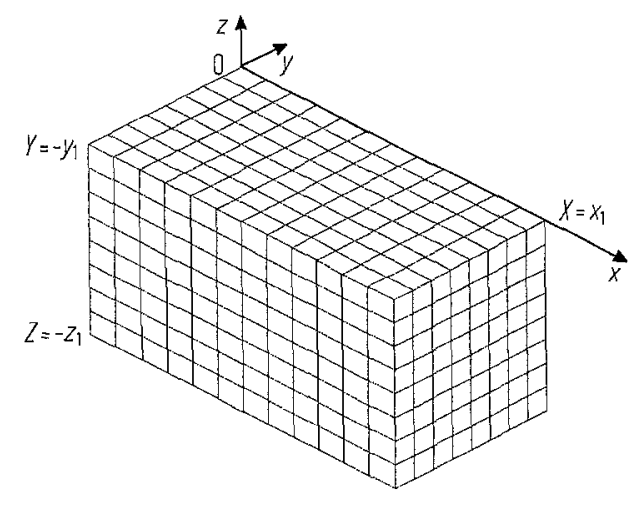

3 
Table 4. CPU-times on a CRAY-1 and a Cray X-MP of the calculation of all singularity contributions for a problem with 416 panels and 1 plane of symmetry

\begin{tabular}{llllll}
\hline method & $\mathrm{A}$ & $\mathrm{B}$ & $\mathrm{C}$ & $\mathrm{D}$ & $\mathrm{E}$ \\
\hline Cray 1 CPU-time (s) & 1.45 & 1.87 & 2.68 & 1.63 & 2.42 \\
Cray X-MP CPU-time (s) & 0.68 & 0.76 & 0.90 & 0.83 & 1.05 \\
\hline
\end{tabular}

For a plane wave moving in the positive $x$-direction the following conditions are used on the artificial boundaries:

$$
\begin{array}{ll}
\frac{\partial \phi}{\partial n}=0 & \text { on } \quad y=y_{1}, \quad y=-y_{1} \\
\frac{\partial \phi}{\partial t}-c \frac{\partial \phi}{\partial n}=0 & \text { on } x=x_{1} \\
\frac{\partial \phi}{\partial t}-c \frac{\partial \phi}{\partial n}=g(y, z, t) & \text { on } \quad x=0
\end{array}
$$

where $g(y, z, t)$ represents the incoming wave with phase-velocity $c$. Starting from a given initial conditions at $t=t_{0}$ the solution procedure consists of discretizing the time-dependent boundary conditions giving an expression for $\phi$ and/or $\frac{\partial \phi}{\partial n}$ at the next time-level $t_{0}+\Delta t$ in a finite number of collocation points. Then the integral equation method is used to solve the boundary value problem obtained at time $t_{0}+\Delta t$, giving the inknown $\phi$ and/or $\frac{\partial \phi}{\partial n}$ on the boundaries. This procedure is repeated each time-step.

As a test example we chose a wave with period $T=2 \pi$ and wave length $\lambda=43.3$. We discretized the boundary as in Fig. 3, involving 416 panels and 1 plane of symmetry. For the time-stepping
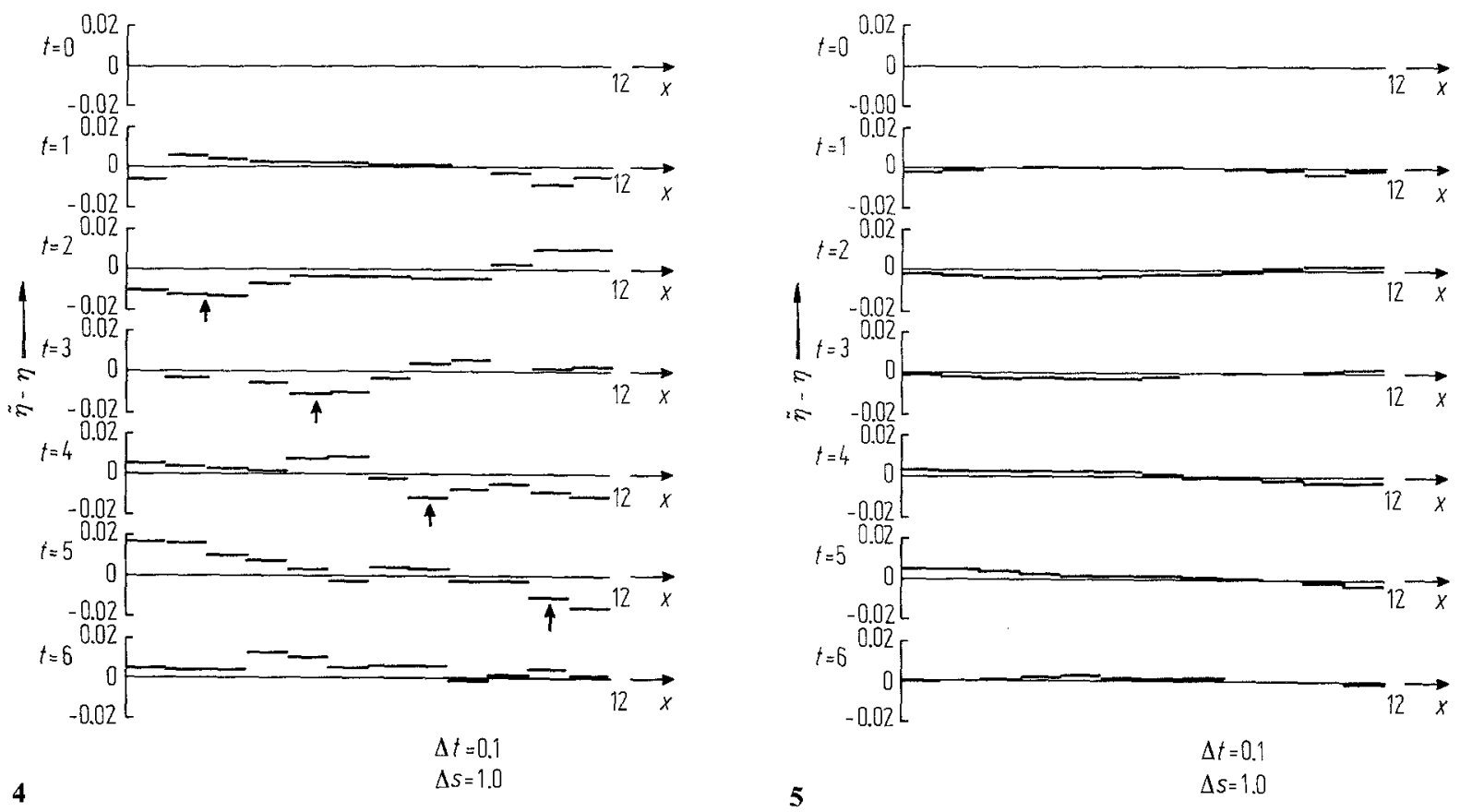

Figs. 4 and 5. 4 The error in the wave elevation $\eta$, along a strip of panels in $x$-direction (option A), shown after $0,10, \ldots$, 50 and 60 time-steps. 5 The error in the wave elevation $\eta$, along a strip of panels in $x$-direction, shown after $0,10, \ldots, 50$ and 60 time-steps; here higher order panels were used at the surface edges 
$(\Delta t=0.1)$ we used a third-order Adams-Moulton scheme. All options in the program performed very well and according to the theoretical results obtained earlier. The method proved to be efficient and reliable. The efficiency of the panel method is illustrated in Table 4, which gives the CPU-times of all methods on a CRAY-1 and a CRAY X-MP supercomputer for the calculation of all sourceand dipole-contributions necessary for the test example. This most time-consuming part of the calculations looks very favourable in comparison with other higher order panel methods. For example, the panel method of NLR (Hoeijmakers 1983) requires $9.5 \times 10^{-4} \mathrm{cpu}$ s per panel-field point combination using an approximation similar to method $\mathrm{E}$ on a Cyber 170-855. For 416 unknowns this would result in $164.4 \mathrm{cpu}$ s. Also using a method similar to $\mathrm{E}$ the program of SaabScania needs $16.1 \mathrm{cpu}$ s on a CRAY-1 for a problem involving 365 unknowns, also with one plane of symmetry ( $\approx 20.8 \mathrm{cpu}$ s for 416 unknowns).

Finally, Fig. 4 illustrates the typical behavior of the error in $\eta$ on the free-surface. It shows the error of 12 consecutive panels in $x$-direction at seven time-levels. Here method A was used on all panels. The errors are introduced at the edges of the free-surface (panels 1 and 12) and penetrate into the interior due to the hyperbolic nature of the free-surface boundary conditions.

To show more clearly that the error, and therefore the accuracy, in the interior of the surface is determined by the errors induced at the surface edges, we repeated the same calculation, but now used a strip of higher order panels (two panels wide) along all the surface edges, to diminish the local error at these edges. Figure 5 shows the results of these computations: the amplitude of the error wave has decreased considerably. This example clearly demonstrates the importance of reducing the error in the corners of the computational domain, since these determine the accuracy of the solution.

\section{Acknowledgements}

The present study was supported by the Netherlands Technology Foundation (STW), by the Dutch Working-Group on Supercomputer Usage (WSG), by Delft Hydraulics, and by the University of Twente.

\section{References}

Hess, J.L. (1975): Consistent velocity and potential expansions for higher-order surface singularity methods. McDonnell Douglas Rep. MDC J6911

Hoeijmakers, H.W.M. (1983): Numerical computation of vortical flow about wings. Nat. Aerospace Laboratory, Amsterdam, Rep. NLR MP 83073 U

Hunt, B. (1978): The panel method for subsonic aerodynamic flows: a survey of mathematical formulations and numerical models, and an outline of the new British Aerospace scheme. VKI Lecture series 1978-4 on Comput. Fluid Dyn.

Longuet-Higgins, M. S.; Cokelet, E.D. (1976): The deformation of steep surface waves on water. I. A numerical method of computation. Proc. R. Soc. London Ser. A 350, 1-26

Romate, J.E. (1988): Local error analysis in 3-D panel methods. J. Eng. Math. 22, 123-142

Vinje, T.; Brevig, P. (1981): Breaking waves on finite water depths. A numerical study. Ship Research Inst., Trondheim, Norway, Rep. R-111.81 